
allemande

50-2 | 2018

Humanités environnementales - Quoi de neuf du côté des méthodes?

\title{
Nachhaltigkeit und Methodik: Verhaltensantriebe und Transformationsbedingungen ermitteln
}

Zugleich zur Findung wirksamer Politikinstrumente mittels multimethodischer qualitativer Governance-Analyse

Felix Ekardt

\section{OpenEdition}

\section{Journals}

Édition électronique

URL : https://journals.openedition.org/allemagne/880

DOI : 10.4000/allemagne.880

ISSN : 2605-7913

Éditeur

Société d'études allemandes

Édition imprimée

Date de publication : 30 décembre 2018

Pagination : 279-296

ISSN : 0035-0974

Référence électronique

Felix Ekardt, „Nachhaltigkeit und Methodik: Verhaltensantriebe und Transformationsbedingungen ermitteln", Revue d'Allemagne et des pays de langue allemande [Online], 50-2 | 2018, Online erschienen am: 30 Dezember 2019, abgerufen am 19 Mai 2021. URL: http://journals.openedition.org/allemagne/ 880 ; DOI: https://doi.org/10.4000/allemagne.880 


\section{Nachhaltigkeit und Methodik: Verhaltensantriebe und Transformationsbedingungen ermitteln}

\section{Zugleich zur Findung wirksamer Politikinstrumente mittels multimethodischer qualitativer Governance-Analyse}

- Felix Ekardt*

\section{Problemstellung}

Wie genau findet man eigentlich heraus, was Menschen antreibt? Wie kann man etwas darüber wissen, was Gesellschaften und Menschen bewegt? Dies ist eine der zentralen, wenn nicht gar die zentrale Frage der Humanwissenschaften, soweit sie sich mit menschlichem Verhalten und ergo mit der menschlichen Interaktion beschäftigen. Thesen und Hypothesen dazu gibt es seit den klassischen Schriften der Weltreligionen, doch wie lässt sich gesichertes Wissen dazu erlangen? Gerade das Streben nach Nachhaltigkeit - also nach dauerhaft und global durchhaltbaren Lebens- und Wirtschaftsweisen - und sein relatives Scheitern gemessen an der Größe der Herausforderungen etwa in puncto Klimawandel, Biodiversitätsverluste, Bodendegradation oder gestörte Stickstoffkreisläufe wirft die Frage auf, wie man menschliches Handeln und eben auch menschliches Untätigbleiben ${ }^{(1)}$ erklären kann. Das betrifft Menschen in Zivilgesellschaft, Politik, Unternehmen oder Nichtregierungsorganisationen

* Prof. Dr. Dr. Felix Ekardt, LL.M., M.A. leitet die Forschungsstelle Nachhaltigkeit und Klimapolitik in Leipzig und Berlin (www.nachhaltigkeit-gerechtigkeit-klima.de) und lehrt Öffentliches Recht und Rechtsphilosophie an der Universität Rostock. Der Verfasser dankt Antonia Sladek, B.A. für Zuarbeiten und Diskussionen speziell zur ethnologischen Perspektive. Der Text entstand innerhalb der Projekte InnoSoilPhos (zum effizienteren Umgang mit Phosphor) und Bioacid (zur Ozeanversauerung), in denen jeweils langjährig große Forschungsverbünde vom BMBF gefördert werden und konkret Governance-Optionen ermittelt werden.

1 Zu diesem mit aktuellen Daten zum ökologischen Fußabdruck, vgl. Umweltbundesamt, Repräsentative Erhebung von Pro-Kopf-Verbräuchen natürlicher Ressourcen in Deutschland (nach Bevölkerungsgruppen), Dessau-Roßlau, 2016 sowie Felix EKARDT, Theorie der Nachhaltigkeit. Ethische, rechtliche, politische und transformative Zugänge - am Beispiel von Klimawandel, Ressourcenknappheit und Welthandel, Baden-Baden, 2016, \$1 B. 
gleichermaßen. Dies ist nicht nur eine inhaltliche Frage danach, wie verschiedene Verhaltensantriebe wie Wissen, Eigennutzenkalküle, Werthaltungen, Gefühle, Normalitätsvorstellungen oder Pfadabhängigkeiten - und hinter allen Faktoren stehend kulturelle (einschließlich ökonomischer) sowie soziobiologische Aspekte - in unserem Verhalten zusammenwirken und prägend wirken ${ }^{(2)}$. Es besteht dem vorgelagert auch die große und schwierige methodische Frage, wie man über menschliche Verhaltensantriebe überhaupt etwas erfahren kann. Diese Frage bildet den Kerngegenstand des vorliegenden Beitrags ${ }^{(3)}$.

Damit verbunden (etwas weniger umfangreich, da zentral auf die erste Frage aufbauend) wird vorliegend einer zweiten Frage nachgegangen: der Frage, wie man methodisch wirksame politisch-rechtliche Maßnahmen für sozialen Wandel identifiziert. Auch dies geschieht am Beispiel von Nachhaltigkeitsfragen, und auch insoweit ist die eigentliche inhaltliche Frage, welche Instrumente dies sein könnten, andernorts öfter Gegenstand gewesen ${ }^{(4)}$. Insoweit geht es kurz gesagt um eine multimethodische qualitative Governance-Analyse. Der Verhaltens- und der Governance-Analyse vorgelagert impliziert der vorliegende Beitrag außerdem einige Klarstellungen zur Erkenntnistheorie auf, die für die beiden anderen Fragen eine Bedeutung haben, aber im allgemeinen Wissenschaftsdiskurs (und auch im Nachhaltigkeitsdiskurs) meist keine oder eine zu hinterfragende Behandlung erfahren; ihnen wird jedoch nur mit einer kurzen Klarstellung nachgegangen. Governance wird vorliegend synonym mit Steuerung gebraucht, also als Frage nach den wirksamen Maßnahmen und konkret politisch-rechtlichen Instrumenten zur Erreichung jeweils vorausgesetzter Ziele (Governance wird hier also nicht - wie gelegentlich - auf spezifisch selbstregulative Vorgänge beschränkt, und auch Good Governance im Sinne eines normativen Systems liberal-demokratischer Grundprinzipien ist vorliegend nicht gemeint ${ }^{(5)}$ ).

Anders als in vielen heutigen Abhandlungen richtet sich der Blick bei der Verhaltensanalyse und auch ansonsten nicht umstandslos auf naturwissenschaftsanalog konzipierte Methoden wie die Befragung (seien sie quantitativ oder qualitativ angelegt) und auch nicht auf Experimente wie etwa jene der Spieltheorie oder auch in Gestalt sogenannter Reallabore oder sogenannter Fallstudien. Vielmehr sollen deren Grenzen thematisiert und ergänzend eine - auch in der Nachhaltigkeitsforschung - weitgehend unbeachtete und auch sonst von vielen verhaltenswissenschaftlichen Disziplinen wie Ökonomik, Psychologie, Soziobiologie, Kulturwissenschaft, Soziologie, Geschichtswissenschaft und Erziehungswissenschaft meist nur am Rande

2 Näher analysiert bei vgl. F. EKARDt, Theorie der Nachhaltigkeit (Anm. 1), 22 sowie Felix EKARDT, Wir können uns ändern. Gesellschaftlicher Wandel jenseits von Kapitalismuskritik und Revolution, München, 2017.

3 Aufbauend auch auf die kurze Erwähnung in vgl. Felix EкARdт, „Suffizienz: Politikinstrumente, Grenzen von Technik und Wachstum und die schwierige Rolle des guten Lebens“, in: Benjamin GöRGen, Matthias Grundmann, Dieter Hoffmeister, Björn Wendt (Hg.), Soziologie und Nachhaltigkeit, 2015, https://www.uni-muenster.de/Ejournals/index.php/sun/article/view/1755.

4 Zusammenfassend vgl. F. ЕкавDт, Theorie der Nachhaltigkeit (Anm. 1).

5 Konfusionen um die Begriffe Steuerung und Governance finden sich bei vgl. Jan Philipp SchAEFER, Die Umgestaltung des Verwaltungsrechts. Kontroversen reformorientierter Verwaltungsrechtswissenschaft, Tübingen, 2016. 
wahrgenommene Methode stärker fokussiert werden. Gemeint ist die teilnehmende Beobachtung, die zwar kaum in den genannten Disziplinen, wohl aber in der Ethnologie und der Religionswissenschaft in den letzten gut hundert Jahren immer wieder zur Anwendung kam, gelegentlich aber auch in anderen Disziplinen wie der Humangeographie, der Entwicklungswissenschaft oder der Sozialen Arbeit ${ }^{(6)}$. Sowohl in der erkenntnistheoretischen Grundlegung als auch in der verhaltenswissenschaftlichen Methodik kommt es damit im Folgenden zu einer Kritik des seit der Aufklärung oft vorherrschenden und vor allem über die Naturwissenschaften konstant perpetuierten Empirismus, der nicht Normen, sondern allein Fakten, und zwar zählbare und reproduzierbare, zum objektiv fassbaren Erkenntnisgegenstand erklärt ${ }^{(7)}$.

Mit einer Kritik am empiristischen - besonders, aber nicht nur in den Naturwissenschaften beliebten - Paradigma kann man mitunter Missverständnisse erzeugen und sich falsche Freunde machen. Hinter einer kritischen Betrachtung empiristischer Perspektiven wird häufig eine postmoderne, aber jedenfalls irgendeine subjektivistische erkenntnistheoretische Position vermutet, die Fakten und Normen für nicht objektiv erkennbar hält. Oder es wird vermutet, man wolle geltend machen, dass humanwissenschaftliche Forschung eben zwangsläufig normativ sei, dass Fakten per se objektiv und Normen per se subjektiv seien - und dass speziell die Nachhaltigkeitsforschung, da sie doch politische Vorschläge unterbreite, zwangsläufig normativ sei. All dies wird hier gerade nicht impliziert ${ }^{(8)}$. Kurz gesagt: Eine Verhaltens- und Nachhaltigkeitsforschung, die sich (unter postmodernen, ethnologischen, feministischen oder noch anderen Vorzeichen) als Kritik der Möglichkeit von Objektivität als vermeintlich bloßes Herrschaftsinstrument verstehen würde, könnte eine Kritik mit allgemeinem Anspruch wieder nur dann formuliert werden, wenn sie selbst Objektivität in Anspruch nimmt und sich damit logisch selbst aufhebt (so wichtig es auch sein dürfte, drohende - menschentypische - Subjektivierungen aufzuspüren, doch genau dieses Aufspüren ist wiederum nur am Maßstab der Objektivität denkbar, weil sonst gar nicht ermittelbar wäre, was „subjektiv“ ist; und generell darf auch nicht die Genese mit der Geltung einer Aussage verwechselt werden). Am Wunsch nach einer „Kritik

6 Bronislaw Malinowski, Argonauts of the Western Pacific. An Account of Native Enterprise and Adventure in the Archipelagoes of Melanesian New Guinea, London, 1932, https://ia802703. us.archive.org/3/items/argonautsofthewe032976mbp/argonautsofthewe032976mbp.pdf; H. Russell Bernard, „Participant Observation“, in: Ders., Research Methods in Anthropology. Qualitative and Quantitative Approaches, London, 1994, S. 136-164; Charlotte Aull DaviEs, Reflexive Ethnography. A guide to researching selves and others, London, 2008; Karen O'Reilly, Ethnographic Methods, London, 2012, S. 25; Antonius Robben, Jeffrey Sluka, Ethnographic Fieldwork. An Anthropological Reader, Malden, 2012.

7 Breiter angelegt dazu vgl. F. Ека Rd , Theorie der Nachhaltigkeit (Anm. 1); Felix Eка dт, Ökonomische Bewertung - Kosten-Nutzen-Analyse - ökonomische Ethik. Eine Kritik am Beispiel Klimaschutzzugleich zu Zahlen im Nachhaltigkeitsdiskurs, Marburg, 2018, widerlegt speziell den Versuch - in Gestalt der Kosten-Nutzen-Analyse respektive ökonomischen Bewertung -, normative respektive Sollensfragen zu Fragen der quantifizierenden Abwägung konkurrierender rein faktischer Präferenzen zu machen. Dieser Ansatz weist bei genauer Betrachtung unlösbare Anwendungsprobleme auf, verfolgt einen ethisch unhaltbaren Grundansatz und kollidiert rechtlich mit den Grundprinzipien liberal-demokratischer Ordnungen.

8 Dagegen und auch zu den folgenden Punkten ausführlich, vgl. F. EKARDT, Theorie der Nachhaltigkeit (Anm. 1). 
der Machtverhältnisse“ ist ferner zu bemerken, dass jene Kritik normative Maßstäbe (die außerdem wieder die Möglichkeit von Objektivität voraussetzen) an den deskriptiven Gegenstand heranträgt und latent beides vermischt.

\section{Teilnehmende Beobachtung: ergänzend zu empiristischen (qualitativen und quantitativen) Analysemethoden der Verhaltensforschung notwendig?}

Selbst wenn man Objektivität in der Faktenfindung grundsätzlich für möglich erachtet, bleibt es methodisch eine große Herausforderung, Verhalten zu erklären, also die Verhaltensantriebe von Bürgern, Politikern, Unternehmern usw. objektiv zu ermitteln (und später darauf dann Governance-Analysen aufzubauen). Unter dem Einfluss insbesondere der Ökonomik, aber auch aktueller Tendenzen in der Psychologie dominiert seit einiger Zeit die Vorstellung, Verhaltensforschung müsste per se naturwissenschaftsanalog vorgehen. Das heißt dann: Forschungsergebnisse sollen reproduzierbar und quantifizierbar sein ${ }^{(9)}$. Um dies zu ermöglichen, führt besonders die Ökonomik spieltheoretische Experimente durch. Darin werden, wie der Name schon sagt, reale Verhaltenssituationen durchgespielt; es wird beispielsweise die klimawandelbezogene Motivation Handelnder durch spielerische Arrangements in Laborsituationen betrachtet, indem man Klimakonferenzen oder alltägliche Konsumentscheidungen nachspielt. Ebenso können auf solch einer Grundlage, ergänzt um viele wirtschafts-, sozial- und naturwissenschaftliche Daten, komplexe Szenarien entstehen, wie es z.B. mit dem Klimaschutz weitergehen könnte. Dagegen versprechen sich viele Forschende der Soziologie und Politologie mehr davon, Leute zu befragen - sei es in großer Zahl mit Fragebögen oder eher mit einer kleinen Zahl von Befragten in ausführlichen, mehr oder minder frei gestalteten Interviews ${ }^{(10)}$.

Der Fokus auf zählbare und reproduzierbare Fakten ist, wie bereits anklang, ein Kind des philosophischen Empirismus seit dem 17. Jahrhundert ${ }^{(11)}$. In Wirklichkeit ist es keineswegs philosophisch selbstverständlich, allein Fakten (und nicht Normen) als Gegenstand rationalen Nachdenkens anzuerkennen, wie bereits zur Sprache kam. Wichtiger für den vorliegenden Beitrag ist jedoch die andere Stoßrichtung einer Kritik am Empirismus: nämlich dass man über menschliches Verhalten und über Wandel durch Experimente und Befragungen möglicherweise zu wenig erfährt.

9 Exemplarisch vgl. Oliver Musshoff, Norbert Hirschauer, „Bereitstellung ökosystemarer Dienstleistungen“, Zeitschrift für Umweltpolitik und Umweltrecht, 2011, S. 437-460; Wolfgang BuchHOLZ, Wolfgang Peters, Aneta Ufert, „Spielräume für uni- und multilateralen Klimaschutz“, Zeitschrift für Umweltpolitik und Umweltrecht, 2014, S. 326-350; kritisch dazu vgl. Christian Schubert, „Mehr Psychologie wagen. Warum eine psychologisch informierte VWL gute Argumente gegen staatlichen Interventionismus liefert“, Wirtschaftliche Freiheit, 2015, http://wirtschaftlichefreiheit.de/ wordpress/?p=1805; Fabian SCheIDler, Das Ende der Megamaschine. Geschichte einer scheiternden Zivilisation, Wien, 2015, S. 106ff.; Ingmar Lippert, „Extended Carbon Cognition as a Machine“, Computational Culture, 2011, http://computationalculture.net/article/extended-carbon-cognition.

10 Experimente mit Wissenschaft weitgehend gleichsetzend, vgl. Guido CANigilia u.a., „Experiments and evidence in sustainability science: A typology“, Journal of Cleaner Production, 2017, S. 1-9.

11 Ausführlich dazu vgl. F. Екавдт, Ökonomische Bewertung (Anm. 7); zur weiteren Kritik von Beobachtungen und Experimenten, schon vgl. F. EKARDT, Theorie der Nachhaltigkeit (Anm. 1), \$1 D. III. 2.; F. EKARDT, Wir können uns ändern (Anm. 2). 
Wenn man wissen will, wie Individuen und Gesellschaften sich wandeln (Verhaltensforschung) und wie Menschen z.B. auf bestimmte neu konzipierte politische Maßnahmen reagieren werden (Governance-Forschung), muss man ihr Verhalten kennen. Diese Kenntnisnahme müsste grundsätzlich in einer Weise ablaufen, dass mit dem Akt der Kenntnisnahme nicht das Verhalten schon verfälscht wird, etwa weil sich die Beobachteten anders zu verhalten beginnen, weil sie sich beobachtet fühlen. Ferner muss man nicht nur das Verhalten an sich, sondern auch seine Motive oder Ursachen erkennen, um tatsächlich Verhalten durch Governance-Optionen beeinflussen zu können und vollständig zu verstehen. Menschliche Motive sind jedoch nicht irgendwo in der äußeren Realität sichtbar. Ebenso ist die Kausalität zwischen Motiven und dem realen Verhalten, auch wenn sie genau wie die Motive zur Welt der Fakten gehört, als solche nicht sichtbar.

Wenn Verhalten und Wandel verstanden werden sollen, wird es deshalb häufig um Rückschlüsse gehen: aus dem Verhalten auf die Motive sowie von Verhalten und Motiven auf die Kausalität. Mit einem philosophischen Begriff kann man das einen Schluss auf die beste Erklärung nennen ${ }^{(12)}$. Ob die Handelnden in einem spieltheoretischen Experiment aus Motiven wie Eigennutzen, altruistischen Werthaltungen, unbewussten Vorstellungen davon, was als normal zu gelten habe oder aus noch anderen Motiven handelt, sieht man dem Verhalten als solchem nicht an. Auch dass beispielsweise Teilnehmende eines Experiments die Option wählen, die ihnen ökonomische Vorteile bringt, zeigt nicht abschließend, dass allein Eigennutzen und bewusste Kalkulation die Motive waren. Es können weitere Motive vorhanden sein. Statistisch ausgedrückt: Die Korrelation von zwei Faktoren muss noch nicht besagen, dass dies die einzigen aufeinander einwirkenden Faktoren sind ${ }^{(13)}$.

Die damit aufgemachten Probleme sind grundsätzlicher Art, und sie gelten für Experimente und Befragungen - ob nun quantitativ von vielen Leuten oder qualitativ in Interviews von wenigen Leuten - im Großen und Ganzen gleichermaßen ${ }^{(14)}$. Ein offenkundiges Problem von Befragungen ist, dass die Antworten das Verhalten und die Motive oft nicht vollständig oder nicht wahrheitsgemäß widerspiegeln - etwa weil

12 Dazu vgl. F. EKardt, Theorie der Nachhaltigkeit (Anm. 1), $\$ 1$ D.II.: Wobei jedoch das Kriterium für „beste“ Erklärung abstrakt ähnlich schwierig zu formulieren ist wie das für „Korrespondenz“ bei der Korrespondenztheorie der Wahrheit, so sehr die Möglichkeit objektiver Wahrheit auch logisch unhintergehbar ist (denn wer die Möglichkeit von Wahrheit bestreitet, kann auch für dieses Bestreiten per se keine Wahrheit in Anspruch nehmen).

13 Vgl. Daniel Отто, Potenziale und Grenzen von epistemic communities. Eine Analyse des Weltklimarates und der Klimarahmenkonvention, Münster, 2015, S. 145ff., übergeht dies, wenn er die mangelhafte Klimapolitik von US-Regierungen allein auf deren Zweifel am Vorliegen eines Klimawandels, also auf die subjektive Überformung von Wissen, zurückführt; unklar auch vgl. Hanjo Hamann, Evidenzbasierte Jurisprudenz. Methoden empirischer Forschung und ihr Erkenntniswert für das Recht am Beispiel des Gesellschaftsrechts, Tübingen, 2014, 142ff.

14 Cum grano salis Matthias MEYER, „Überlegungen zur Rationalität institutionenökonomischer Modelle“, in: Anne van Aaken, Stefanie Schmid-Lübbert (Hg.), Beiträge zur ökonomischen Theorie im Öffentlichen Recht, Wiesbaden, 2003, S. 149-170; H. Hamann, Evidenzbasierte Jurisprudenz (Anm. 13), S. 250f; F. Scheidler, Das Ende der Megamaschine (Anm. 9), S. 106ff.; F. EkARdT, Theorie der Nachhaltigkeit (Anm. 1), \$1 D. III. 2.; unberücksichtigt bei vgl. Daniel LANG, Horst Rode, Henrik von WeHrden, „Methoden und Methodologie in den Nachhaltigkeitswissenschaften“, in: Harald Heinrichs, Gerd Michelsen (Hg.), Nachhaltigkeitswissenschaften, Heidelberg, 2014, S. 115-135. 
das eigene Handeln und seine sozialen Bedingungen nicht immer bewusst reflektiert werden können ${ }^{(15)}$. Dazu kommen weitere verfälschende Faktoren wie der Wunsch, den Interviewenden zu gefallen, die Erwartungen zu treffen und mit sozialen Konventionen in Übereinstimmung zu bleiben. Auch die Art der Fragen und der Gesprächskontext präformieren häufig die möglichen Antworten. Geht es in einer Befragung etwa um Umweltschutz, wird dieser damit von vornherein als relevant und sozial erwünscht gelabelt. Allein schon durch dieses aktive Thematisieren einer Frage werden Verhalten und Motive in erheblicher Weise überformt - die Leute erscheinen dadurch, salopp gesagt, mehr öko, als sie tatsächlich sind. Solche Probleme kann man durch die Befragungstechnik minimieren, aber kaum ausschalten. Außerdem ziehen bei der Frage nach Motiven und bei der Vielfalt von alltäglichen Einzelhandlungen vom Essen über die Fortbewegung bis hin zum Wohnen, die nachhaltigkeitsbezogen relevant wären, deren Komplexität und mögliche (zeitweiliger oder ständiger) Unbewusstheit des jeweiligen Motivs deutliche Grenzen ${ }^{(16)}$. Es kann ferner Fehlvorstellungen über das eigene Verhalten und dessen Motive geben, die auf emotionalen Mechanismen wie Verdrängung beruhen ${ }^{(17)}$ : Zufriedenheit mit der Ehe war größer bei regelmäßig befragten Paaren als bei seltener Interviewten; Abtreibung wurde eher gegenüber der Interviewerin als dem Interviewer angesprochen. Insofern kann man die in der Umweltsoziologie gängigen Studien ${ }^{(18)}$ als etwas optimistisch empfinden, wenn sie Umweltbewusstsein durch Befragung ermitteln wollen - also Fakten und Werthaltungen, womit sonstige weniger greifbare Motive unter den Tisch fallen (und selbst die Aussagen zu Werthaltungen aus den diversen aufgelisteten Gründen sehr mit Vorsicht zu genießen sind) - und ggf. auch das Verhalten der Probanden auf gleiche Weise erheben wollen.

Erhebt man Befragungen streng quantitativ, macht man also echte Statistiken, kommen weitere verfälschende Faktoren hinzu. So ist die Zufallsauswahl der Befragten oft nicht repräsentativ. Zudem sind bestimmte Bevölkerungsgruppen (vor allem Ältere) leichter über Anrufe auf dem Festnetztelefon erreichbar als andere. Und auch vorangegangene Befragungen beeinflussen die Äußerungen, etwa wenn die Befragten wissen, dass zuletzt ein bestimmtes Ergebnis bei einer ähnlichen Befragung erzielt wurde. Selbst wenn trotz all dieser Faktoren - was bereits recht unwahrscheinlich sein dürfte - die Befragten weitestgehend wahrheitsgemäß antwortet, stellt sich das zusätzliche Problem des Auseinanderfallens von Einstellung und Verhalten. Befragungen können direkt Verhalten und seine (ggf. vorgeblichen oder vermeintlichen) Motive erfragen, sie können aber auch Einstellungen wie etwa die Haltung zum Umweltschutz erfragen und

15 Udo Kelle, „Integration qualitativer und quantitativer Methoden“, in: Udo Kuckartz u.a. (Hg.), Qualitative Datenanalyse, Wiesbaden, 2007, S. 63; F. Екаврт, Theorie der Nachhaltigkeit (Anm. 1), $\$ 1$ D. III. 2 .

$16 \mathrm{Zu}$ aktuellen psychologischen Debatten des Unbewussten, vgl. auch Matthias Kettner, Wolfgang Mertens, Reflexionen über das Unbewusste. Philosophie und Psychologie im Dialog, Göttingen, 2010, S. 7ff., 77ff. und 109ff.

$17 \mathrm{Zu}$ den verschiedenen Friktionen, vgl. auch Raymond M. LEE, Unobtrusive methods in social research, Buckingham, 2000; F. EKardT, Theorie der Nachhaltigkeit (Anm. 1), \$\$1 D. III. 2., 2 C.-D.

18 Wie etwa vgl. Dieter Hoffmeister, Björn Wendt, Luigi Droste, Nachhaltigkeit in Münster. Studierende und Normalbürger - Ressource für eine zukunftsfähige Stadtentwicklung?, Münster, 2014. 
daraus das mögliche Verhalten gegenüber einer stärkeren Umweltpolitik abzuleiten versuchen. Letzteres scheitert wegen des gerade umweltbezogenen, letztlich aber allgemeinmenschlichen Gaps zwischen Einstellung und Verhalten und oftmals auch zwischen verschiedenen gleichzeitig vorhandenen, sich aber widersprechenden Einstellungen ${ }^{(19)}$.

Dennoch müssen die damit erheblichen und nicht grundsätzlich ausräumbaren Friktionen nicht zwangsläufig zu der Konsequenz führen, sich der Methode der quantitativen oder qualitativen Befragung gar nicht mehr zu bedienen. Es kann z.B. Fragen geben, bei denen die Neigung gering ist, einer Interviewer- oder Gesellschaftserwartung nachzugeben. Zudem haben Befragungen gerade in quantitativer Ausführung das Potenzial, strukturelle Zusammenhänge und breite Meinungsspektren zu adressieren. Im Lichte der beschriebenen Probleme erscheinen sie jedoch primär dann interessant, wenn sie mit weiteren, nachstehend näher zu entwickelnden Zugängen kombiniert werden. Bevor dem nachgegangen wird, ist jedoch auf den zweiten verbreiteten empiristischen Methodenansatz einzugehen.

Im Grundsatz gelten die zu Befragungen vorgetragenen Einwände nämlich auch für Experimente, mögen es die genannten spieltheoretischen Anordnungen oder sogenannte Real-World Laboratories sein wie etwa das Durchspielen eines ressourcenarmen Lebensstils für eine gewisse Zeit im realen Leben ${ }^{(20)}$. So beeinflussen auch hier soziale Erwünschtheiten und die Anwesenheit von Beobachtern die Versuchspersonen, was sich bereits bei den sogenannten Hawthrone-Studien in den 1930er Jahren zeigte: Die Teilnehmenden verbesserten ihre Arbeitsleistung nicht wie angenommen aufgrund von veränderten Lichtbedingungen, sondern wegen der Anwesenheit der Beobachtenden ${ }^{(21)}$. Hinzu kommt, dass die Übersetzung der meist hochkomplexen Wirklichkeit (in puncto Ausgangssituation und Handlungsoptionen) in ein notgedrungen reduktionistisches Experiment kaum gelingen kann; dieses weist außerdem einen fiktiven Charakter auf. Man stelle sich z.B. eine spieltheoretische Situation vor, in der die hochkomplexen globalen Klimaverhandlungen nachgespielt werden ${ }^{(22)}$. Weder können die sozialen Erwünschtheiten und Beobachtererwartungen ausgeblendet werden, noch kann das

19 Zum Umgang mit kognitiven Dissonanzen näher, vgl. Susanne Stoll-KleEmann, Tim O’Riordan, Carlo JAEGER, „The psychology of denial concerning climate mitigation measures: evidence from Swiss focus groups", Global Environmental Change, 2001, S. 107ff.; Weyma Lüвве, Verantwortung in komplexen kulturellen Prozessen, Freiburg, 1998; Felix EKardT, Steuerungsdefizite im Umweltrecht. Ursachen unter besonderer Berücksichtigung des Naturschutzrechts und der Grundrechte - zugleich zur Relevanz religiösen Säkularisats im öffentlichen Recht, Sinzheim, 2001, § 13. 3.; F. ЕKARDT, Theorie der Nachhaltigkeit (Anm. 1), \$2 D.

20 Matthias Gross, Holger Hoffmann-Riem, Wolfgang Krohn, Realexperimente. Ökologische Gestaltungsprozesse in der Wissensgesellschaft, Bielefeld, 2005, S. 19ff.; kaum problematisiert bei vgl. Martin Nowak, Roger Highfield, Kooperative Intelligenz. Das Erfolgsgeheimnis der Evolution, München, 2013, S. $225 \mathrm{ff}$.

21 R. LeE, Unobtrusive methods (Anm. 17), S. 5.

22 Kritisch vgl. Paula KivimaA u. a., „Experiments in Climate Governance - Lessons from a Systematic Review of Case Studies in Transition Research", SPRU Working Paper Series, Sussex, 2015, S. 2ff.; affirmativ vgl. Manfred Milinski, Jochem Marotzke, „Das Klimaspiel. Warum scheitern Klimaverhandlungen?", in: Jochem Marotzke, Martin Stratmann (Hg.), Die Zukunft des Klimas. Neue Erkenntnisse, neue Herausforderungen, München, S. 93-110; siehe auch vgl. Sebastian BAmBerg, "Changing environmentally harmful behaviors: A stage model of self-regulated behavioral change", Journal of Environmental Psychology, 2016, S. 151-160. 
Empfinden der Spieler in einer solchen Konstellation einfach mit der Situation real Handelnder gleichgesetzt werden, noch erfährt man durch das Beobachten von - zudem auch noch fiktiven - Spielzügen überhaupt direkt etwas über deren Motive. Diese muss man vielmehr erneut aus ihren - fiktiven - Handlungen erschließen.

Zwar kann man vermeintlich naturwissenschaftsanalog einzelne experimentelle Bedingungen variieren und dadurch den Einfluss einzelner Faktoren herauszuarbeiten versuchen. Doch weder Erwünschtheiten noch fiktiver Charakter noch Unterkomplexität verschwinden dadurch. Hinsichtlich des fiktiven Charakters ist es z.B. so, dass in der Realität meist eine derartige Vielzahl von Faktoren auf eine Entscheidung einwirken, dass dies nicht sinnvoll auf eine Versuchssituation heruntergebrochen werden kann, dies durch beispielsweise nur drei Faktoren bestimmt ist. Auch wenn man das Experiment - als Reallabor - ins wirkliche Leben verlegt, erledigt sich keines dieser Probleme. Zwar wird der fiktive Charakter dann etwas geringer. Dennoch bleibt es auch dann etwas grundlegend anderes, ob man nur einmal einen Monat so tut, als würde man ressourcenleicht leben, und zwar unter aufmunternder Beobachtung ökologisch eingestellter Wissenschaftler - oder ob dies tatsächlich dauerhaft so ist. Spätestens bei Reallaboren, letztlich aber auch bei Experimenten zeigt sich außerdem, dass es um die unter empiristischen Vorzeichen angestrebte beliebige Replizierbarkeit humanwissenschaftlicher Experimente deutlich schlechter bestellt ist als in den Naturwissenschaften (erst recht gilt das für qualitative Ansätze).

Abschließend $\mathrm{zu}$ alledem ein weiteres Beispiel (was hier, da die entsprechenden Versuche noch nicht veröffentlicht sind, nur allgemein umschrieben sei). Der Verfasser bringt regelmäßig seine Söhne zur Teilnahme an Kleinkindexperimenten an verschiedenen Leipziger Forschungseinrichtungen ${ }^{(23)}$. Einem aktuellen verhaltenswissenschaftlichen Dauerthema folgend, wird dort insbesondere untersucht, wie kooperativ sich Kleinkinder in bestimmten Situationen verhalten. Dafür werden die Kinder beispielsweise in Ballspiele verwickelt, anhand derer sich dann zeigen soll, wie sehr Menschen miteinander oder gegeneinander agieren. Doch was beweisen solche Befunde wirklich: Ist ein Befund zur Kooperation in einem Ballspiel wirklich geeignet, den Umfang menschlicher Kooperativität in realen - und weit komplexeren und meist nicht spielerischen - realen Lebenssituationen zu bestimmen? Und was würde man über die Motive der Kooperation erfahren - ob diese etwa dem Beobachter gefallen soll, eigennützigen Vorteilskalkülen oder vielmehr altruistischen Fairnesserwägungen geschuldet ist? Oder ob sie schlicht Normalitätsvorstellungen geschuldet ist, die der Versuchsperson nicht bewusst sind und in ihrem Umfeld einfach unhinterfragt geteilt werden? Oder ob emotionale Faktoren wie Empathie, der Wunsch nach Anerkennung, Bequemlichkeit oder Gewohnheit eine Rolle spielen ${ }^{(24)}$ ? Nichts davon erfährt man wirklich über entsprechende Experimente. Genau solche Informationen wären aber wesentlich, wenn man z.B. im Zuge der Transformation zur Nachhaltigkeit erfahren will, was die Transformation bisher bremst - und auf welche Reaktion neue politische Maßnahmen rechnen müssten. 
Wie schon bei Befragungen bedeutet auch das zuletzt Gesagte nicht, dass Experimente keine Hinweise auf Verhalten, seine Ursachen und damit die Bedingungen von Wandel geben könnten. Gleichwohl benötigt man aufgrund der genannten verzerrenden Effekte weitere Erkenntnisquellen. Vergleichsweise hoch ist ihre Tauglichkeit einzuschätzen, wenn das Setting so gewählt ist, dass das eigentliche Experiment vor den Probanden weitgehend verborgen bleibt wie etwa beim berühmten MilgramExperiment zur Gehorsamsbereitschaft gegenüber - vermeintlichen - Autoritäten ${ }^{(25)}$.

Die geschilderten Friktionen werden nicht nur in vielen Fachdiskursen mehr oder minder übergangen oder zumindest so behandelt, als würden sie Befragungen und Experimenten keine substanziellen Grenzen setzen ${ }^{(26)}$. Sie lösen sich auch nicht, wenn man die aufgeworfenen Methodenfragen der Verhaltensforschung dadurch $\mathrm{zu}$ erledigen versucht, dass man wie der wirtschaftswissenschaftliche Mainstream ein einfaches Verhaltensmodell wie den Homo oeconomicus postuliert, also einen stets bewusst kalkulierenden und rein nutzenorientierten - und zwar normalerweise eigennutzenorientierten - Menschen unterstellt. Dieses Modell wird schon in der Ökonomik selbst als unterkomplex erkannt, etwa im Rahmen der Behavioral-Economics-Forschung ${ }^{(27)}$, auch wenn das Modell gleichzeitig immer weiter Verwendung findet ${ }^{(28)}$. Doch ein unterkomplexes Modell kann keine methodisch gesicherte Verhaltensermittlung ersetzen.

Wenn also Verhalten schwer zu erfassen ist und erst recht die Motive und Kausalitäten sich primär interpretativ erschließen, erfährt man Wesentliches über Motive, Wandel und oft schon über das Verhalten selbst nur über eine andere, weniger formale Erkenntnisquelle. Gemeint ist die einleitend bereits erwähnte teilnehmende Beobachtung im Sinne einer möglichst umfangreichen Fremdbeobachtung. Dabei wird aus dem Verhalten auf Motive rückgeschlossen und damit auf die genannten, verfälschenden Faktoren, die in der beschriebenen Weise eine quantifizierbare Erkenntnis behindern, reagiert. Gerade bei Nachhaltigkeitsfragen spricht einiges dafür, dass man ohne möglichst unbemerkte - und möglichst häufige und umfassende - Beobachtung nicht auskommt. Denn besonders in diesem gesellschaftlichen Bereich pflegen viele ein sehr umweltfreundliches Selbstbild, welches eben leider mit einem unverändert großen ökologischen Fußabdruck scharf kontrastiert ${ }^{(29)}$. Dem ist jetzt in den beiden folgenden Abschnitten weiter nachzugehen, einschließlich der Frage nach eventuell nötigen weiteren Methoden oder Kontrollschritten.

25 Stanley Milgram, Das Milgram-Experiment. Zur Gehorsamsbereitschaft gegenüber Autorität, Reinbek, 1982.

26 Exemplarisch die Beiträge in vgl. Ulrike Oster maier (Hg.), Hochbegabung - Exzellenz-Werte. Positionen in der schulischen Begabtenförderung, Dresden, 2011.

27 Zusammenfassend dargestellt bei vgl. F. EKARDT, Ökonomische Bewertung (Anm. 7).

$28 \mathrm{Zu}$ den starken Orthodoxie-Tendenzen in der Ökonomik, vgl. Hartmut Rosa u.a., Weil Kapitalismus sich ändern muss, Wiesbaden, 2014, S. 41ff; Albert LöHr, Eckhard BURKATZKI, „Resozialisierung der ökonomischen Rationalität - thematische Einführung“, Jahrbuch Ökonomie und Gesellschaft, 2015, S. 11-50.

29 Zum empirischen Befund, vgl. F. Ека Rт, Theorie der Nachhaltigkeit (Anm. 1), \$2 B. 


\section{Teilnehmende Beobachtung, Formalisierungsgrade, Kontrollmechanismen und Wege zu einer integrierten Methodik}

Teilnehmende Beobachtung meint gemäß dem Methoden-Lexikon für die Sozialwissenschaften $^{(30)}$ ein Beobachtungsverfahren, bei dem der oder die Beobachtende sich selbst aktiv an dem Geschehen beteiligt, das beobachtet wird, wobei er ggf. persönlich bemerkt, häufig aber nicht als gezielter Beobachter bemerkt wird. Die Rede ist damit von einem Zugang, der alltäglich und unbemerkt praktiziert werden kann, sich aber auch stärker an Experimente und Befragungen anlehnen kann, indem man einen klar abgegrenzten Vorgang beobachtet, häufig wiederkehrend, und seine Beobachtereigenschaft ggf. auch offenlegt. Konzentriert man sich auf äußerlich beobachtbare Handlungen, führt dies demgemäß zu stärker formalisierten Settings, die dann allerdings wieder vor der Frage stehen, inwieweit das formalisierte Erfassen einiger äußerer Zusammenhänge wirklich etwas über die als solche stets verborgene Motivationslage der Beobachteten aussagt - auf die es in der Verhaltensforschung (s.o.) ja gerade ankommt. Da gewissermaßen jede Person an ihren sozialen Interaktionen mindestens informell beobachtend teilnimmt ${ }^{(31)}$, lässt sich diese Form der Erkenntnisgewinnung auch als ein Kontinuum beschreiben, das bei einfachen Alltagsbeobachtungen beginnt und bis zu mehrjährigen ethnographischen Beobachtungsstudien reicht. Es kann also ein systematisches Forschungsdesign vorliegen, es kann aber auch wesentlich weniger formell vorgegangen werden. Die beobachtende Person ist vor Ort, nimmt das Geschehen also in seinem natürlichen Umfeld und mithilfe aller Sinne wahr. Die teilnehmende Beobachtung kategorisiert ihren Gegenstand und hält in irgendeiner Weise ihre Ergebnisse fest, wobei das mehr oder weniger systematisch erfolgen kann ${ }^{(32)}$.

Es kann sich dabei auch um echte zufällige Alltagsbeobachtungen wie z.B. das Kaufverhalten und Gespräche in Lebensmittelgeschäften, aber auch Selbstbeobachtungen wie etwa Gedankengänge beim eigenen Einkauf handeln. Ziel ist eine detaillierte, tiefgehende Beschreibung und Interpretation von sozialen Phänomenen mit Rücksicht auf ihre Vielschichtigkeit und Widersprüchlichkeit ${ }^{(33)}$. Dabei beschränken sich solche Felder nicht wie ursprünglich in der Ethnologie auf tribale oder bäuerliche Gemeinschaften; stattdessen kann jedes vorstellbare kulturelle Setting ethnographisch

30 Rainer Diaz-Bone, Christoph Weischer, Methoden-Lexikon für die Sozialwissenschaften, Heidelberg, 2015, S. 40, http://link.springer.com/book/10.1007\%2F978-3-531-18889-8; siehe auch vgl. Georg Breidenstein u.a., Ethnografie. Die Praxis der Feldforschung, Konstanz, 2015.

31 Vgl. Kathleen M. DeWalt, Billie DeWalt, Participant Observation. A Guide for Fieldworkers, Walnut Creek, 2002, S. 1, beschreiben dies am Beispiel des Versuchs, in einer Ansammlung von Menschen Anschluss zu finden, denen man fremd ist und die sich alle zu kennen oder zumindest zu verstehen scheinen. Um sich an ihren Gesprächen zu beteiligen, wird man zunächst ihr äußeres Erscheinungsbild und Auftreten beobachten und versuchen, die Themen und z.B. den Grad der Formalität ihrer Konversationen herauszuhören.

32 Siehe einerseits vgl. G. Breidenstein u.a., Ethnografie (Anm. 30); und andererseits vgl. Bettina Beer, "Systematische Beobachtung“, in: DIEs. (Hg.), Methoden und Techniken der Feldforschung, Berlin, 2003, S. 129ff.

33 G. Breidenstein u.a., Ethnografie (Anm. 30), S. 8f.; Wolfgang Kaschuba, Einführung in die Europäische Ethnologie, München, 2012, S. 208. 
erkundet werden ${ }^{(34)}$. Die auch als Ethnographien ${ }^{(35)}$ bezeichneten umfassenden Beobachtungen, die es in der einen oder anderen Form schon seit der Antike gibt ${ }^{(36)}$, wollen soziales Handeln so untersuchen, wie es sich in seiner alltäglichen Umgebung, in konkreten Kontexten entfaltet ${ }^{(37)}$. Übergreifende Intention im Sinne möglichst authentischer Ergebnisse ist es also, Verhalten nicht unter Laborbedingungen, sondern in den „natürlichen“ Handlungskontexten zu beobachten ${ }^{(38)}$.

Die Chancen dieses Zugangs werden an einem Beispiel noch besser verständlich ${ }^{(39)}$. Erfragt man bei Menschen Werthaltungen in puncto Nachhaltigkeit oder führt man dazu Experimente durch, so zeigen diese regelmäßig starke ökologische Werthaltungen und auch einen guten Fakten-Informationsstand zum Thema. Gleichzeitig zeigt der aus der gesamten ökologischen Belastungssituation statistisch rückschließbare ökologische Fußabdruck pro Kopf, dass das reale Verhalten nicht zu diesen Einstellungen passt. Das könnte heißen, dass die Befragungen und Experimente zu unwahren Ergebnissen führen, weil die Probanden dem Interviewer oder vermuteten sozialen Erwartungen gerecht werden wollen, Nachhaltigkeit also in Wirklichkeit unwichtig finden. Kumulativ oder alternativ könnte es aber auch sein, dass die Probanden durchaus teils wahrheitsgemäß antworten, aber schlicht eine sehr viel komplexere Motivationslage haben, die sich bei Befragungen und Experimenten nicht zeigt. Dies kann man einer Klärung näherbringen, wenn man in einer Vielzahl von Alltagsgesprächen - ohne besondere Aktivierung einer sozialen Erwartung und ohne künstlich herbeigeführte Situation darauf achtet, wie Menschen sich zu Nachhaltigkeitsfragen positionieren. Dabei wird z.B. ein klarer Eigennutzenfokus - neben allen durchaus vorhandenen Werthaltungen pro Nachhaltigkeit - sichtbar. Gleichzeitig zeigen sich Pfadabhängigkeiten und Kollektivgutprobleme - es besteht also der Eindruck, man könne nicht anders, als man sich real verhalte, und außerdem sei der eigene Beitrag zu einem globalen Problem ohnehin belanglos. Analoge Befunde kann man durch Selbstbeobachtung und durch Rückschlüsse aus der biologischen Herkunft des Menschen, also aus soziobiologischen Analysen, gewinnen, die darauf beruhen, dass Menschen einerseits egoistisch,

34 DeWalt/DeWalt, Participant Observation (Anm. 31), S. 1.

35 Griech. ethnos (Volk) und graphein (schreiben); bezeichnet die fertige Monographie und zugleich die Feldforschung selbst als den dominanten Forschungsmodus der klassischen Ethnologie; vgl. Brigitta Schmidt-LAuber, „Feldforschung. Kulturanalyse durch teilnehmende Beobachtung“, in: Silke Götтsch, Albrecht Lehmann (Hg.), Methoden der Volkskunde. Positionen, Quellen, Arbeitsweisen der Europäischen Ethnologie, Berlin, S. 221. Der eigentliche Wortsinn wird dem heutigen Gebrauch der Methode nicht mehr gerecht.

36 Beschreibungen von Bräuchen, Eigenarten, Fähigkeiten „fremder“ Kulturen finden sich schon bei Herodot, in der römische Antike, im Kaiserreich China und auch in den ab dem 7. Jh. aufsteigenden islamischen Reichen sowie bei Missionaren und Händlern aus Europa ab dem 13. Jh.; vgl. Rosalie H. WAx, Doing Fieldwork. Warning and Advice, Chicago, 1985, S. 21ff. Im Zuge des Imperialismus ab dem späteren 19. Jahrhundert stieg die Zahl solcher Berichte über „fremde“ Kulturen rapide an.

37 K. O’Reilly, Ethnographic Methods (Anm. 6), S. 3; Danny L. Jorgensen, Participant Observation. A Methodology for Human Studies, London, 1989, S. 12.

38 Tom Boellstorff u.a., Ethnography and Virtual Worlds: A Handbook of Method, New Jersey, 2012, S. 38.

39 Näher zu empirischen Nachweisen für das Folgende, vgl. F. EкARDT, Theorie der Nachhaltigkeit (Anm. 1), \$2 und F. EKARDT, Wir können uns ändern (Anm. 2); die hier herangezogenen inhaltlichen Befunde zur Nachhaltigkeits-Motivation werden hier nur sehr knapp wiederholt, da vorliegend der Fokus auf der Methodik liegt. 
andererseits auch kooperativ agieren, allerdings auch letzteres oft mit eigennützigen oder zumindest "gruppenegoistischem“ Hintergrund. Dass vor diesem Hintergrund eine Orientierung an Werten im sozialen Nahbereich oft vorkommt, gleichzeitig aber dann an Grenzen stößt, wenn Klimaschutz im Interesse „der Menschheit“ gefragt ist, passt zu diesen Befunden. Deckungsgleich lässt sich in Befragungen, Experimenten und teilnehmenden Beobachtungen ferner sehen, dass Menschen, deren eigenes Leben in puncto Nachhaltigkeit hinterfragt wird, ihren Lebensstil meist für unabänderlich erklären und mit Abwehr oder sogar Aggression reagieren. Ferner lässt sich beobachten (passend zu statistischen Daten in puncto Umweltschutz), dass auch ökonomisch eigennützige Handlungsoptionen - etwa Gebäudewärmedämmung, wenn das Geld dafür vorhanden ist - oftmals nicht gezogen werden. Dies macht deutlich (wiederum unterstützt durch Selbstbeobachtung sowie evolutionsbiologische Befunde zu menschlichen Strategien im Umgang mit einer überkomplexen Welt), dass Nachhaltigkeitshandeln und überhaupt menschliches Verhalten keinesfalls immer bewusst kalkuliert eigennützig oder altruistisch ist. Vielmehr sind auch Emotionen im Spiel wie Bequemlichkeit, Gewohnheit, Verdrängung oder die Fähigkeit, selbst offenkundige Widersprüche von Reden und Handeln beiseite zu schieben (was sich wiederum experimentell ebenfalls bestätigt). Daneben scheint es einen Faktor Normalitätsvorstellungen zu geben, der ähnlich wie Emotionen - und anders als Werthaltungen oder Eigennutzenkalküle den Handelnden oft nur bedingt bewusst ist: Hoher Fleischkonsum, Urlaubsflüge und die tägliche Autofahrt zur Arbeit sind in Industriestaaten (und bei den Oberschichten der Schwellenländer) heute schlicht „normal“. Sehr greifbar wird das, wenn eine Vielzahl Beobachteter auf Hinweise, man selber esse z.B. kein Fleisch, fahre kein Auto usw., dies schlicht amüsant, eben irgendwie „unnormal“ findet ${ }^{(40)}$. Was von den jeweils (hier sehr kurz) geschilderten Antrieben kulturell geprägt und was vielleicht schon genetisch im Menschen angelegt ist, kann man u.a. anhand dessen untersuchen, ob bestimmte

40 Weder Gewohnheiten noch Normalitätsvorstellungen müssen irgendwann bewusst angenommen worden sein. Vielmehr dürfte es sich um einen schleichenden Prozess handeln, wobei gerade die Sozialisation in der Kindheit und in der jeweiligen Kultur eine wichtige Rolle spielt. Jeder Mensch erwirbt die kulturellen Faktoren und aktiviert die biologischen Faktoren nach allem, was wir wissen, im Rahmen seines persönlichen Werdegangs, der soziologisch auch als Sozialisation bezeichnet werden kann. Vorliegend werden die Motivationselemente indes bewusst nicht von der Sozialisation her aufgeschlüsselt, weil in diesem Konzept Vorstellungen davon mitschwingen, dass es um die Genese des jeweils unverwechselbaren Individuums und um das Entstehen einer geteilten Welt mit anderen gehe. Redet man so, belastet man sich u.a. mit erkenntnistheoretischen und neurophysiologischen Fragen danach, ob es „das“ Individuum im Sinne eines festen Selbst so überhaupt gibt. Ausführlich dazu vgl. Yuval Noah Harari, Homo Deus. Eine Geschichte von Morgen, München, 2017. Ferner drohen in der betonten Rede von der Sozialisation unterschwellige normative Kategorien mitzuwirken (etwa dass es wünschenswert ist, sich zu entwickeln, eigene Erfahrungen zu machen usw.), die zu einer deskriptiven Verhaltenstheorie wenig beitragen können. Zentral zur Sozialisationstheorie dagegen, vgl. Matthias Grundmann, Norm und Konstruktion. Zur Dialektik von Bildungsvererbung und Bildungsaneignung, Opladen, 1998; DERS., Sozialisation. Skizze einer allgemeinen Theorie, Konstanz, 2006; zur normativen Seite, vgl. DERs., „Gemeinsam - nachhaltig. Argumente für eine sozialisationstheoretische Bestimmung sozialer Nachhaltigkeit“, Soziologie und Nachhaltigkeit, 2016, https://www.uni-muenster.de/ Ejournals/index.php/sun/article/view/1756/1690. Davon versucht sich der vorliegende Zugang freizumachen, ebenso wie von ebenfalls normativ imprägniert wirkenden Begrifflichkeiten wie primäre versus sekundäre Motivationssysteme, dafür aber vgl. Stefan BRunnhuber, Die Kunst der Transformation. Wie wirlernen, die Welt zu verändern, Freiburg, 2016. 
Faktoren weltweit oder nur regional auftreten. Jedenfalls zeigt sich mit alledem, dass teilnehmende Beobachtung gerade in Anwendung auf das häufige - nicht nur für den Nachhaltigkeitskontext charakteristische - Auseinanderfallen von Einstellungen, sozialen Erwartungen und realem Verhalten erhebliche Einsichten verspricht; dies wurde auch in nicht nachhaltigkeitsbezogenen Kontexten bereits bemerkt ${ }^{(41)}$.

Gleichzeitig wurde bereits deutlich, dass gerade die beschriebene Kombination von teilnehmender Beobachtung mit anderen Ansätzen wie Befragungen, Experimenten, Selbstbeobachtung oder soziobiologischen Ableitungen notwendig und sinnvoll im Sinne einer möglichst kritischen wechselseitigen Prüfung aller Befunde erscheint. Eine formalisierte, den Beobachteten offiziell mitgeteilte teilnehmende Beobachtung dürfte z.B. teils ähnliche Verzerrungseffekte wie die empiristischen Forschungsmethoden auslösen ${ }^{(42)}$. Genau diese wurde soeben allerdings auch weniger empfohlen. Selbst bei einer verdeckten teilnehmenden Beobachtung problematisch sind indes das eigene begrenzte Wissen, subjektive Perspektiv-Verengungen, die oft übertrieben positive Wahrnehmung der eigenen Person, die Beschränkungen der eigenen Sicht über den sozialen Hintergrund usw. ${ }^{(43)}$. Dazu kommt, dass die teilnehmende Beobachtung nur schwer die gesellschaftliche Verteilung von sozialen Phänomenen erfassen kann, wie dies groß angelegte quantitative Studien vermögen. Übersehen werden diese Grenzen, wenn Verhaltens- oder Gesellschaftsforschung etwa allein auf „Milieukenntnis“ im Luhmannschen Sinne gestützt werden würde ${ }^{(44)}$. Weiterführend erscheint also gerade die Kombination der teilnehmenden Beobachtung mit Befunden aus Experimenten, Befragungen und soziobiologischen Ableitungen - und zunehmend auch den Befunden neurowissenschaftlicher und biochemischer Forschung, laufen im menschlichen Nervensystem doch diverse messbare elektrische oder stoffliche Prozesse bei Entscheidungen $\mathrm{ab}^{(45)}$. Auch letztere Zugänge unterliegen freilich - gerade beim aktuellen Forschungsstand - noch vielerlei Grenzen ${ }^{(46)}$, was die Notwendigkeit wechselseitiger Überprüfung Prüfungen bekräftigt.

41 B. Beer, „Systematische Beobachtung“ (Anm. 32), S. 126; G. Breidenstein u.a., Ethnografie (Anm. 30); Franz Schultheis, „Nachwort“, in: Pierre Bourdieu, Ein soziologischer Selbstversuch, Frankfurt am Main, 2002, S. 133-151; Liz STAnLEY, „Mass-Observation's Fieldwork Methods“, in: Paul Atkinson u.a. (Hg.), Handbook of Ethnography, London, 2013, S. 92-108.

42 Brigitta Hauser-Schäublin, „Teilnehmende Beobachtung“, in: Bettina BeER (Hg.), Methoden ethnologischer Feldforschung, Berlin, 2008, S. 37-58.

43 K. O’Reilly, Ethnographic Methods (Anm. 6), S. 96; Martyn Hammersley, Paul Atkinson, Ethnography. Principles in Practice, London, 2007, S. 15; B. Hauser-Sch ÄUblin, "Teilnehmende Beobachtung“ (Anm. 42), S. 54; Jörg NiewöHner u.a., „Einleitung“, in: Ders. u.a. (Hg.), Science and Technology Studies. Eine sozialanthropologische Einführung, Bielefeld, 2012, S. $13 \mathrm{f}$.

44 Alexander KrAfFT, „Wie Niklas Luhmann die Welt beobachtet“, in: Institut für Soziologie und Sozialforschung der Carl von Ossietzky-Universität Oldenburg (Hg.), Der soziologische Blick. Vergangene und gegenwärtige Perspektiven, Opladen, 2002, S. 197.

45 Yuval Noah Harari, Eine kurze Geschichte der Menschheit, München, 2013; Ders., Homo Deus (Anm. 40).

46 Kritisch dazu vgl. Felix HASLER, Neuromythologie. Eine Streitschrift gegen die Deutungsmacht der Hirnforschung, Bielefeld, 2012; F. EкARDT, Theorie der Nachhaltigkeit (Anm. 1), \$2 E.; siehe auch vgl. Stephan PoHL, Wissenschaftstheoretische und methodologische Probleme der Psychoanalyse. Eine Auseinandersetzung mit Grünbaums Psychoanalysekritik, Würzburg, 1991, S. IVf. und T. Boellstorff u.a., Ethnography (Anm. 38), S. 29ff., gegen vermeintlich rein naturwissenschaftliche Zugänge. 
Bereits die geschilderte Methodenkombination dient der Vermeidung oder Verminderung eines subjektiv reduzierten Blickwinkels sowie einer zu geringen Breite der teilnehmenden Beobachtung. Daneben muss die teilnehmende Beobachtung als solche möglichst akkurat ausgeführt werden. Sie setzt zunächst voraus, dass die Forschenden über einen längeren Zeitraum Zugang zur einschlägigen Alltagswelt haben und an den routinierten wie auch außeralltäglichen Tätigkeiten und Vorgängen teilnehmen ${ }^{(47)}$. Die langfristige Präsenz im Feld ist essentiell, um sich die „fremden“ Sichtweisen und Routinen zunehmend anzueignen und sich dabei von den „eigenen“ Vorannahmen zu lösen ${ }^{(48)}$. Doch das „Eintauchen“ in eine Kultur, die intensive körperliche und seelische Teilnahme also, muss stets mit der distanzierten Beobachtung konterkariert werden ${ }^{(49)}$. Um diese Dialektik der teilnehmenden Beobachtung aufrecht zu erhalten, wird von verschiedenen Techniken der Beobachtungsintensivierung sowie von analytischen Abstraktionsverfahren Gebrauch gemacht ${ }^{(50)}$. Ein mögliches Hilfsmittel ist das Forschungstagebuch, worin die Interaktionen im Feld systematisch in Form von Feldnotizen dokumentiert werden, aber auch das Sammeln von Artefakten wie Tonband- und Videoaufnahmen oder Dokumenten ${ }^{(51)}$. Damit spielen Reflexion und Selbstbeobachtung eine wesentliche ergänzende Rolle. Insoweit gilt es auch, sich Sachverhalte wie begrenztes Wissen und die übertrieben positive Wahrnehmung der eigenen Person bewusst zu machen und sie daher mindestens stark zu relativieren, insbesondere auch im Diskurs mit anderen ${ }^{(52)}$.

Noch eine weitere methodische Ergänzung zu den beschriebenen Zugängen erscheint angezeigt. Sie besteht in der Berücksichtigung des Umstands, dass äußere (etwa geographische und technische) und politisch-rechtliche Rahmenbedingungen das Verhalten z.B. von Konsumierenden oder Unternehmen naheliegenderweise beeinflussen. Damit kommt neben Naturgegebenheiten auch die wechselseitige Beeinflussung der

47 Zum Ganzen vgl. Jean JACKson, „Participant Observation“, in: Thomas Barfield (Hg.), The Dictionary of Anthropology, Malden, 2002, S. 348; DeWalt/DeWalt, Participant Observation (Anm. 31), S. 4; Jean Schensul, Margaret LeCompte, The ethnographer's toolkit, 3. Essential ethnographic methods: a mixed methods approach, Walnut Creek, 2013, S. 83; Miriam CoHN, „Teilnehmende Beobachtung", in: Christine Bischoff u.a. (Hg.), Methoden der Kulturanthropologie, Stuttgart, 2014, S. 72; Diaz-Bone/Weischer, Methoden-Lexikon (Anm. 30), S. 41; H. R. Bernard, „Participant Observation“ (Anm. 6).

48 K. O’Reilly, Ethnographic Methods (Anm. 6), S. 92 und 94; H. R. Bernard, „Participant Observation“ (Anm. 6), S. 137; W. KAschuBA, Einführung (Anm. 33), S. 207.

49 K. O’Reilly, Ethnographic Methods (Anm. 6), S. 106; J. JACKson, „Participant Observation“ (Anm. 47), S. 348; B. Schmidt-Lauber, „Feldforschung“ (Anm. 35), S. 220; B. Hauser-Schäublin, „Teilnehmende Beobachtung“ (Anm. 42), S. 41; G. Breidenstein u.a., Ethnografie (Anm. 30), S. 7; Diaz-Bone/Weischer, Methoden-Lexikon (Anm. 30), S. 41.

50 Umfassend dargestellt werden diese Verfahren etwa bei vgl. G. Breidenstein u.a., Ethnografie (Anm. 30).

51 G. Breidenstein u.a., Ethnografie (Anm. 30), S. 71; B. Hauser-Schäublin, „Teilnehmende Beobachtung“ (Anm. 42), S. 53; Hammersley/Atkinson, Ethnography (Anm. 43), S. 3; R. Lee, Unobtrusive methods (Anm. 17); D. L. Jorgensen, Participant Observation (Anm. 37), S. 22; H. R. Bernard, „Participant Observation“ (Anm. 6), S. 142; Michael Angrosino, Doing Ethnographic and Observational Research, London, 2007, S. 61.

52 U. Kelle, „Integration“ (Anm. 15); Ines Steinke, „Gütekriterien qualitativer Forschung“, in: Uwe Flick (Hg.), Qualitative Forschung. Ein Handbuch, Reinbek, 2000, S. 322ff. 
Handelnden ins Spiel; denn hinter „der Politik“ beispielsweise verbergen sich wieder Menschen. Die mit alledem mögliche breite Absicherung von Analysen ${ }^{(53)}$ erreicht man z.B. allein mit Experimenten nicht. Wenn man beispielsweise ${ }^{(54)}$ in Experimenten den Befund erhält, dass in einer alläglichen - nicht nachhaltigkeitsbezogenen - Situation Betroffene ihren Dreck lieber selbst wegmachen, macht dies gerade nicht plausibel, dass Menschen rundum gerne für die Folgen ihres Handelns einstehen und sich deshalb etwa nach einer massiven Klimapolitik sehnen. Dass dies so nicht sein kann, ergibt sich schon daraus, dass etwa das Klimaproblem bisher nicht wirksam angegangen wird, obwohl wir jenes Problem täglich durch unseren ganz normalen Lebensstil weiter perpetuieren.

\section{Aus Verhaltensanalysen Steuerungsprobleme und Steuerungsinstrumente ableiten - zugleich zu Grenzen von Zahlen im Nachhaltigkeitsdiskurs}

Der kombinierte respektive triangulierte Ansatz zur Analyse menschlicher Verhaltensmotive ergibt nicht nur eine Analyse der Ursachen von Nicht-Nachhaltigkeit respektive der Bedingungen einer Transformation zur Nachhaltigkeit ${ }^{(55)}$. Wie einleitend bereits angedeutet, kann darauf eine multimethodische qualitative Governance-Analyse im Sinne einer Suche nach wirksamen Maßnahmen und konkret politisch-rechtlichen Instrumenten zur Erreichung jeweils vorausgesetzter Ziele aufgebaut werden. Dazu vorliegend nur in aller Kürze ${ }^{(56)}$ :

Zunächst einmal können wie gesehen menschliche Verhaltensweisen und gerade auch Verhaltensmotive multimethodisch ermittelt werden. Dies ist eine unverzichtbare Basis, um die Wirksamkeit von Governance-Instrumenten abzuschätzen, insbesondere im (für Nachhaltigkeitsfragen typischen) Fall, dass Instrumente entwickelt werden, die es in der Realität so noch nicht - oder unter anderen Bedingungen - gegeben hat. Folgerichtig muss das Verhalten der jeweiligen Steuerungsadressaten anhand von Erkenntnissen über deren Motivationslage antizipiert werden. Zu dieser Abschätzung können wie gesehen auch Befragungen und Experimente, wie sie Ökonomen gern praktizieren, beitragen (etwa zu Preiselastizitäten bei den Adressaten), die aber ihre oben ausführlich dargelegten Grenzen haben; und insbesondere reicht es nicht

$53 \mathrm{Zu}$ Bürgern, Politikern, Unternehmern usw. und ihren Wechselwirkungen - dazu und zur Ablehnung des Analysierens mit unpersönlichen „Strukturen“, vgl. F. EKARDT, Theorie der Nachhaltigkeit (Anm. 1), §2 A.

54 Siehe Michael Јаков u.a., „Clean up your own mess: An experimental study of moral responsibility and efficiency“, Journal of Public Economics, 2017, 10.1016/j.jpubeco.2017.09.010.

55 Dazu, dass es nicht sinnvoll erscheint, vermeintliche „Strukturen“ als etwas jenseits der hier vorgestellten Analyse liegendes „Zusätzliches“ zu betrachten, vgl. F. ЕкARDT, Theorie der Nachhaltigkeit (Anm. 1), §2 A.

56 Der vorliegende Ansatz entstand in ersten Grundzügen - methodisch und inhaltlich - während der Dissertation des Verfassers, vgl. F. ЕкаR D, Steuerungsdefizite (Anm. 19), zu den Ursachen von NichtNachhaltigkeit, wobei damals der kulturelle Faktor Protestantismus besonders intensiv beleuchtet wurde. Damals ging die Untersuchung von einem Blick auf die Vielzahl an Disziplinen, die über Umweltverhalten schon einmal nachgedacht hatten - und auf eine Analyse, was dabei für Fehler gemacht wurden. Ausgehend davon wurde damals erstmals ein übergreifender Ansatz formuliert, der seitdem aber erheblich weiterentwickelt wurde, zuletzt vgl. DERs., Theorie der Nachhaltigkeit (Anm. 1); DERS., Wir können uns ändern (Anm. 2). 
aus, wie der ökonomische Mainstream mit der Spieltheorie vom rein eigennützig und ständig bewusst-kalkulierend agierenden Adressaten auszugehen. Insofern muss hier der dargelegte multimethodische Zugang zur Verhaltensforschung greifen.

Die so auffindbaren Verhaltensmotive ${ }^{(57)}$ bilden eine Basis, um bestimmte erwartbare Steuerungsprobleme als in hohem Maße erwartbar $\mathrm{zu}$ plausibilisieren ${ }^{(58)}$. In Nachhaltigkeitsfragen sind dies insbesondere Rebound-Effekte (einschließlich Wohlstandseffekten), räumliche/ sektorale/ von einem zum anderen Umweltproblem hin stattfindende Verlagerungseffekte, Abbildbarkeitsprobleme, mangelnde Zielstrenge und Vollzugsprobleme. Die Existenz ebenjener Steuerungsprobleme kann man, da es wie gesagt oft um so noch nicht dagewesene Steuerungskonstellationen geht (etwa um eine vollständige Dekarbonisierung innerhalb weniger Jahre), nicht einfach in der Realität beobachten; ergo ist Verhaltensforschung als Basis nötig. Gleichwohl spielen weitere empirische Einsichten neben der Verhaltensforschung für die weitere Erhärtung eine wichtige Rolle. Dass gesamtwirtschaftlich z.B. Treibhausgasemissionen verlagert werden, kann man in Ansätzen (wenngleich unter großen Schwierigkeiten) durchaus messen, indem man die Treibhausgasintensität von Produkten aufgrund technischer Daten bestimmt und sie sodann mit statistischen Im- und Exportdaten kombiniert ${ }^{(59)}$. Beim Rebound-Effekt ist das freilich schon schwieriger, weil die Kausalität zwischen den diversen einzelnen Aspekten schwer dingfest zu machen ist ${ }^{(60)}$. Der somit nötige verhaltenswissenschaftliche Zugang zu Steuerungsproblemen ist sogar bei der Prüfung der Effektivität real praktizierter Steuerungsoptionen wichtig, denn selbst wenn sie existieren, ist oft schwer zu beantworten, welche gesellschaftlichen Entwicklungen wirklich just dem zu prüfenden Steuerungsinstrument zuzuschreiben sind.

Die Hinweise eben zu den Steuerungsproblemen zeigen, dass ergänzend Faktoren wie offenkundige Eigenarten der Instrumente und weitere naturwissenschaftliche, technische und ökonomische Gegebenheiten einen wesentlichen Beitrag dazu leisten, bestimmte Instrumente als voraussichtlich effektiv oder ineffektiv zu identifizieren. Es spricht jedoch vieles dafür, die damit skizzierte multimethodische GovernanceAnalyse qualitativ durchzuführen und auf scheinexakte Quantifizierungen relativ weitgehend zu verzichten. Denn allein schon die Verhaltensmotive und auf ihnen aufbauend die Steuerungsprobleme lassen sich nicht umfassend und exakt, sondern nur punktuell quantifizieren. Dann kann mit ihnen aber auch nicht gerechnet werden bzw. nur unter Inkaufnahme des Problems gerechnet werden, dass eine Vielzahl von Annahmen getroffen wird, die so nicht zutreffen müssen. Dabei können nicht einmal sinnvoll Wahrscheinlichkeiten für das Auftreten bestimmter Faktoren hieb- und stichfest mathematisiert werden, weil ebenjene Wahrscheinlichkeiten in aller Regel nicht bekannt sein werden; dies vereitelt dann jedoch Berechnungen. Das Gleiche gilt

57 Siehe kurz oben und ausführlich m.w.N, vgl. DERs., Theorie der Nachhaltigkeit (Anm. 1), \$2; DERS., Wir können uns ändern (Anm. 2).

58 Ebd.

59 Glen Peters u.a., Growth in emission transfers via international trade from 1990 to 2008, 2011, PNAS, S. 8903-8920.

60 Zur Diskussion, vgl. Tilman Santarius, Der Rebound-Effekt. Ökonomische, psychische und soziale Herausforderungen für die Entkopplung von Wirtschaftswachstum und Energieverbrauch, Marburg, 2015. 
für die naturwissenschaftlichen, technischen und ökonomischen sonstigen Befunde. Erschwerend kommen jeweils unklare Ursachenzusammenhänge zwischen verschiedenen Faktoren und gerade in Nachhaltigkeitsfragen der letztlich globale Referenzrahmen hinzu ${ }^{(61)}$.

Mit alledem ergeben sich abschließend zwei weitere Implikationen, gerade auch für das Verhältnis zu den Naturwissenschaften. Erstens stößt die Zahlenfixierung des empiristischen Paradigmas auf vielfältige Grenzen. Denn, ohne dass dies hier aus Raumgründen im Detail ausgeführt werden kann: Nicht nur Verhalten ist nicht zählbar. Auch Biodiv-/ Ökobilanz-/ Szenarien-Fakten sind dies weithin nicht ${ }^{(62)}$. Noch weniger kann ${ }^{(63)}$ eine normative Rechtfertigung von Nachhaltigkeit durch eine vermeintlich alles quantifizierende, also zählbar machende, Kosten-Nutzen-Analyse ersetzt werden. Auch die verbreitete Suche nach vermeintlich empirisch abgeleiteten, in Wirklichkeit aber normativ gemeinten (doch in aller Regel nicht wirklich rechtlich oder ethisch begründeten) Nachhaltigkeitsindikatoren, die wiederum der Quantifizierungslogik verpflichtet sind, wirft insoweit vielfältige Fragen auf.

Zweitens bleibt die Erkenntnisfindung in Nachhaltigkeitsfragen durch die große Herausforderung Transdisziplinarität geprägt. Gemeint ist mit Transdisziplinarität hier ein Denken von Fragestellungen und nicht von Disziplingrenzen oder gar Schulen her, welches demgemäß eine große Menge an Zugängen und Argumenten verarbeiten muss ${ }^{(64)}$. Zitierbare Literatur findet man zwar für so ziemlich jede denkbare These, gerade in der Verhaltensforschung, wobei die jeweiligen Forschungsrichtungen häufig gewisse autopoetische Selbstbestätigungstendenzen - gesichert durch das notorische Beiseitelassen anderer Disziplinen, Schulen und Erkenntnisse - zeigen ${ }^{(65)}$. So gesehen ist es nicht weiter zu rechtfertigen, dass die verschiedenen Verhaltenswissenschaften sich oftmals weitgehend gegenseitig ignorieren. Vorbehalte bei den meisten Soziologen etwa gegen Soziobiologen, Neurologen und Ökonomen bedürfen daher dringend der Überprüfung - umgekehrt allerdings auch. Legt man die Erkenntnisse verschiedener Disziplinen übereinander, trianguliert man Methoden und prüft sie damit kritisch, könnte das tatsächlich interessante Ergebnisse ermöglichen. Dass das an eine

61 Ausführlich und kritisch zu alledem, vgl. Christian Dieckноғ;, Modellierte Zukunft. Energieszenarien in der wissenschaftlichen Politikberatung, Bielefeld, 2015; DERs., Anna Leuschner (Hg.), Die Energiewende und ihre Modelle. Was uns Energieszenarien sagen können - und was nicht, Bielefeld, 2016; Felix Ekardt, Anika Zorn, Jutta Wieding, „In 10 Jahren globale Nullemissionen? Widersprüche zwischen Art. 2 und 4 Paris-Abkommen und ihre Auflösung. Zugleich zu Vorsorgeprinzip, vagen Bezugsgrößen und überschätzten Klimaszenarien“, Momentum Quarterly, i.E., 2018; exemplarisch für einen berechnungsoptimistischen Zugang, vgl. Benjamin BoDIRsky u.a., „Global Food Demand Scenarios for the $21^{\text {st }}$ Century“, PLOS ONE, 2015, DOI:10.1371.

$62 \mathrm{Zu}$ letzteren wieder, vgl. C. Dieckhoff, Modellierte Zukunft (Anm. 61); Ders./Leuschner (Hg.), Die Energiewende (Anm. 61); EKARdT/Zorn/Wieding, „In 10 Jahren“ (Anm. 61); zu ersteren, vgl. Felix EkARdt, Bettina HenNig, Ökonomische Instrumente und Bewertungen der Biodiversität. Lehren für den Naturschutz aus dem Klimaschutz?, Marburg, 2015.

63 Näher dazu, vgl. F. EKARDT, Ökonomische Bewertung (Anm. 7).

64 Dazu auch, vgl. Matthias Bergmann u.a., Methoden transdisziplinärer Forschung, Frankfurt am Main, 2010.

65 Zum Problem gerade der menschlichen Vereinfachungsneigung auch in wissenschaftlichen Kreisen, vgl. Felix Eка дт, Kurzschluss. Wie einfache Wahrheiten die Demokratie untergraben, Berlin, 2017. 
Überforderung der einzelnen Wissenschaftler grenzen kann, dürfte zutreffen - zumal man in Nachhaltigkeitsfragen gut daran tut, auch die naturwissenschaftlichen Ausgangspunkte wie Szenarien mit ihren Tausenden von Hintergrundannahmen nicht einfach pauschal zu "glauben“. Vermeidbar erscheint diese Zumutung letzten Endes aber nicht.

\section{Zusammenfassung}

Was Menschen (im Alltag wie auch im gesellschaftlichen Zusammenwirken) antreibt und was damit auch sozialen Wandel auslöst, beispielsweise hin zur Nachhaltigkeit, ist eine Kernfrage der (auch Umwelt-)Humanwissenschaften. Seit langem ist kontrovers, mit welchen Methoden sich - eben nicht direkt beobachtbare - menschliche Verhaltensmotive erheben lassen. Der vorliegende Beitrag entwickelt in Abgrenzung vom auf Befragungen und Experimente hin ausgelegten empiristischen Paradigma eine multimethodische qualitative Verhaltensanalyse, in der Befragungen und Experimente (einerlei ob quantitativ oder qualitativ) nur noch zwei Bausteine von mehreren im Rahmen einer breiter angelegten Methodenkombination sind. Mit alledem wird zugleich eine wesentliche Methodenfrage einer Transformation zur Nachhaltigkeit behandelt. Darauf aufbauend wird vorliegend ein Schema für eine qualitative Analyse von Governance-Instrumenten (wie umweltökonomischen Instrumenten oder Ordnungsrecht) angeboten. Insgesamt wird zugleich sichtbar, welche Implikationen eine allzu naturwissenschaftsanaloge Ausrichtung der Umwelt-Humanwissenschaften hat - und welchen Möglichkeiten und Grenzen Quantifizierungen respektive Zahlen im Umweltdiskurs unterliegen. 